\title{
Treatment of patellar dislocation with arthroscopic medial patellofemoral ligament reconstruction using gracilis tendon autograft and modified double- patellar tunnel technique: minimum 5-year patient-reported outcomes
}

\author{
Guanying Gao, Ping Liu* and Yan Xu*
}

\begin{abstract}
Background: The purpose of this study was to retrospectively analyze the clinical outcomes of patients with recurrent patellar dislocation who underwent arthroscopic medial patellofemoral ligament (MPFL) reconstruction using gracilis tendon autograft and a modified double-patellar tunnel method. We hypothesized that our modified method would provide good clinical outcomes.

Methods: Patients who underwent arthroscopic MPFL reconstruction with autograft gracilis tendon and modified double-patellar tunnels technique for recurrent patellar dislocation and were followed up for a minimum of 5 years were identified, and the clinical and follow-up data were retrospectively analyzed. Preoperatively, joint hypermobility was assessed with the Beighton score. The Insall-Salvati ratio, TT-TG distance, and Q angle were measured on radiographic images. Patient-reported outcomes including the Kujala, Lysholm, and Tegner scores were collected preoperatively and postoperatively. Patient satisfaction was assessed at the end of 5 years. Complications and recurrent dislocation occurring after surgery were recorded.
\end{abstract}

Results: A total of 79 patients (94 knees) were enrolled; of these, 13 (16.5\%) were lost to follow-up. The data of 66 patients (80 knees) were available for final analysis. Mean age at surgery was $21.3 \pm 7.8$ years. Mean follow-up time was $66.1 \pm 5.5$ months (range, 60-78 months). Postoperative patient-reported outcome was not associated with Beighton score, Insall-Salvati ratio, or T-TG distance. Q angle was negatively correlated to Kujala scores and Lysholm scores. Severity of trochlear dysplasia was not associated with postoperative patient-reported outcome. The mean Kujala score increased from $69.4 \pm 7.9$ to $96.1 \pm 1.9$, the mean Tegner score increased from $3.1 \pm 1.3$ to $5.9 \pm 1.3$, and the mean Lysholm score increased from $73.5 \pm 14.6$ to $95.3 \pm 3.4$. Two patients experienced recurrent patellar dislocation during follow-up.

Conclusions: MPFL reconstruction using autologous gracilis tendon under arthroscopy appears to be a reliable and safe method for treating recurrent patellar dislocation.

Level of evidence: Level IV.

Keywords: Medial patellofemoral ligament, Reconstruction, Recurrent patellar dislocation, Patient-reported outcomes

\footnotetext{
* Correspondence: bjusmlp@126.com; yanxu@139.com

Institute of Sports Medicine, Peking University Third Hospital, 49 North Garden Road, Haidian District, Beijing 100191, China
}

(c) The Author(s). 2020 Open Access This article is distributed under the terms of the Creative Commons Attribution 4.0 International License (http://creativecommons.org/licenses/by/4.0/), which permits unrestricted use, distribution, and reproduction in any medium, provided you give appropriate credit to the original author(s) and the source, provide a link to the Creative Commons license, and indicate if changes were made. The Creative Commons Public Domain Dedication waiver (http://creativecommons.org/publicdomain/zero/1.0/) applies to the data made available in this article, unless otherwise stated. 


\section{Introduction}

Acute patellar dislocation is a common injury, especially among adolescents, and is usually related to sports and physical activities [1]. It may be triggered by traumatic or nontraumatic events [2]. About 15-44\% of patients who are treated conservatively after an acute dislocation will have recurrent patellar dislocation [3, 4]. Patients with anatomic variations such as a large $Q$ angle, patella alta, femoral trochlear dysplasia, and arthrochalasis are particularly prone to recurrence [5-8].

There are numerous surgical options available to treat patellar dislocation but no consensus as yet on which one is the most effective. Medial patellofemoral ligament (MPFL) reconstruction can provide excellent clinical results and has been increasingly applied in recent years [9-11]. The MPFL is composed of an inferior-straight bundle at the medial aspect of the patella and a superior-oblique bundle at the superiormedial aspect of patella [12]. Because of this doublebundle structure of the MPFL, a patellar doubletunnel approach is recommended to achieve anatomical reconstruction [13, 14]. The conventional method of MPFL reconstruction usually requires two incisions: one at the patellar inner margin and another at the femoral medial epicondyle $[9,15,16]$. Because MPFL is located in the second layer, incision of the extensor apparatus is also necessary. For the patients in this study, we used endoscopic fenestration of the articular capsule to expose the medial patellar bone margin and then drilled two bone tunnels under arthroscopy to reconstruct the MPFL.

Risk factors for patellar dislocation include increased Q angle, patella alta, excessive TT-TG (tibial tubercle-trochlear groove) distance, trochlear dysplasia, and arthrochalasis. Some scholars recommend that these factors also be corrected during surgery. For example, tibial tubercle osteotomy is recommended in patients with TT-TG distance $>20 \mathrm{~mm}$ [17-19], as an excessively large TT-TG increases patellar lateral stress and results in lateral dislocation. Frequent dislocation leads to increased medial patellar joint pressure, which induces patellofemoral joint degeneration [20].

The purpose of this study was to evaluate outcomes in patients with recurrent patellar dislocation treated with arthroscopic MPFL reconstruction using autograft gracilis tendon and a modified double-patellar tunnel method. Patient-reported outcomes were evaluated after 5-year follow-up. We hypothesized that MPFL reconstruction using autologous gracilis tendon under arthroscopy would provide good clinical outcomes and Beighton score, Insall-Salvati ratio, TTTG distance, and $\mathrm{Q}$ angle would be correlated to patient-reported clinical outcomes.

\section{Methods}

\section{Patients}

A total of 79 patients who underwent MPFL reconstruction using autograft gracilis tendon with modified two patellar tunnels technique between January 2012 and June 2013 were included in this retrospective study. All patients had been operated upon by the same surgeon. Patients were eligible for inclusion if (1) they had had patellar dislocation at least twice, and (2) they had been followed up for at least 5 years after the surgery. Patients with bony defects, concomitant ligament injury, or prior knee surgery were excluded from this study. The clinical, operative, and follow-up data were collected from the hospital records and analyzed retrospectively.

This study was approved by The Ethics Committee of the Third Hospital of Peking University our institutional review board (IRB 000067612014205). All participants signed informed consent before surgery.

\section{Functional and radiographic evaluation}

Standard anterolateral radiograph of the knee in $30^{\circ}$ flexion, Merchant-view radiographs, and knee joint CT were obtained before surgery. Preoperatively, the Beighton score and Q angle [21] were measured and recorded for all patients. The Beighton score was used to evaluate joint hypermobility by functional examination. The Insall-Salvati ratio was calculated according to the method of Insall and Salvati [22] and trochlear dysplasia was evaluated (according to the method of Dejourat et al. [17]) on a standard lateral radiograph of the knee in $30^{\circ}$ flexion. The TT-TG distance was measured on the knee joint CT [17]. Insall-Salvati ratio, TT-TG distance, and $\mathrm{Q}$ angle were measured by the treating surgeon (who is also the corresponding author). The Beighton score was measured by the first author. We have planned to follow-up all patients with imaging, but only parts of patients (60.6\%) were followed up with postoperative imaging because some patients refused.

\section{Surgery technique}

Arthroscopy was performed first to treat any intraarticular synovitis, cartilage injury, loose bodies, and other such problems. Then, the fascia was exposed, and the gracilis tendon was harvested using a tendon stripper. The graft diameter was about $3-3.5 \mathrm{~mm}$. The cut ends of the tendon were sutured together with braided suture. A capsular window was made at the medial cartilage margin of the patella under arthroscopy to expose the medial border of the patella. The patellar tunnels were prepared arthroscopically. An auxiliary approach was established about $3 \mathrm{~cm}$ medial to the midpoint of the inner edge of the patella as the working portal and the procedure of preparing patellar tunnel was viewed from medial arthroscopic approach. Two bone tunnels 
(3.5-mm diameter) were drilled at the superomedial half of the patella toward the patellar surface under arthroscopy until the cortex of patellar was penetrated. The two exits of the bone tunnels were located in the medial part of the midline of the patella surface. A distance of about $1 \mathrm{~cm}$ between tunnels was maintained to avoid fracturing the patella (Fig. 1a). The midpoint between the femoral medial epicondyle and the adductor tubercle was identified by palpation, and a $1-\mathrm{cm}$ longitudinal incision was made. At this point, which is the anatomic termination point of the MPFL [23, 24], a femoral bone tunnel with a diameter of $5 \mathrm{~mm}$ was drilled under direct vision. A subcutaneous pathway was established between the longitudinal incision and the medial margin of the patella by blunt penetration. The gracilis tendon, along with a guide wire, was passed through the two patellar bone tunnels, forming U-type loop. The two ends of the tendon were drawn between the medial part of the second and third joint capsule layers to the femoral bone tunnel through the subcutaneous pathway. Then, with the knee flexed to $90^{\circ}$, the two ends of the graft were fixed in the femoral bone tunnel using a bioresorbable interference screw (Fig. 1b) [18, 25-27]. Finally, the surgeon confirmed under arthroscopy that the patellar tracking was appropriate and that the knee joint could be freely flexed to $110^{\circ}$.

\section{Postoperative rehabilitation}

A hinged brace locked in extension was worn for 4 weeks after surgery. Partial weight-bearing exercise was started at 2 weeks after surgery and full weight-bearing exercise at 4 weeks after surgery. Range of motion exercise began 5 days after surgery, with $90^{\circ}$ flexion being achieved by the postoperative second week. The degree of flexion was gradually increased and, on average, normal range of motion was achieved by 2 months after surgery. Controlled sports activities such as jogging were allowed at 4 months after surgery, and full return to sports was allowed at 6 months. Postoperative rehabilitation was guided by the same physical therapist.

\section{Outcome scores}

The Kujala, Lysholm, and Tegner scores of all patients were evaluated before and after operation. Patient satisfaction with final outcome (graded as excellent, good, or fair) was documented at the end of 5 years. Complications and dislocations occurring after surgery were recorded.

\section{Statistical analysis}

The paired $t$ test (two-tailed) was used to evaluate differences between preoperative and postoperative assessments. Pearson correlation analysis was used to evaluate the relationship between postoperative patient-reported outcomes and Beighton score, Insall-Salvati ratio, Q angle, and TT-TG distance. SPSS10.0 software (SPSS Inc., Chicago, IL, USA) was used for statistical analysis. Statistical significance was at $P<.05$.

\section{Results}

Of the 79 patients (94 knees) initially included in the study, 13 (16.5\%) patients (14/94 knees; $14.9 \%)$ were lost to follow-up. Thus, the data of 66 patients ( 80 knees) were available for final analysis. These 66 patients included 17 men and 49 women. Table 1 summarizes the demographic characteristics of the study patients. Mean age at the time of surgery was $21.3 \pm 7.8$ years (range, 13-50 years). Mean body mass index (BMI) was 22.5 (range, 18.3-27.7). Mean interval from initial injury to operation was $6.2 \pm 10.1$ months (range, 0.2-30 months). Mean postoperative follow-up was for $66.1 \pm$ 5.5 months (range, 60-78 months). The clinical follow-

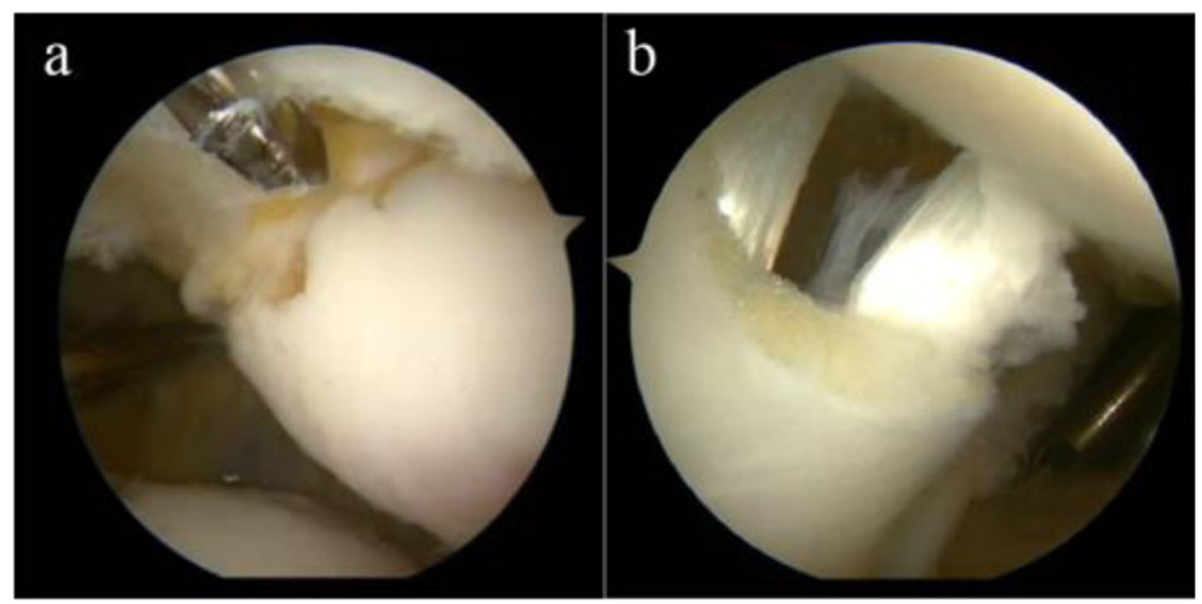

Fig. 1 a Preparation of the patellar tunnel under arthroscopy. b Reconstructed MPFL at arthroscopy 
Table 1 Patient demographics

\begin{tabular}{ll}
\hline & Value \\
\hline Number of patients & 66 \\
Number of knees & 80 \\
Follow-up rate, \% & 83.5 \\
Mean age at surgery, years & $21.3 \pm 7.8(13-50)$ \\
BMl, $\mathrm{kg} / \mathrm{m}^{2}$ & $22.5(18.3-27.7)$ \\
Duration of symptoms, months & $6.2 \pm 10.1(0.2-30)$ \\
Gender (F/M), $n$ & $49 / 17$ \\
Side (L/R), $n$ & $40 / 40$ \\
Follow-up period, months & $66.1 \pm 5.5(60-78)$ \\
Number of recurrent patellar dislocations & 2 \\
\hline
\end{tabular}

Values are shown as mean \pm SD (range) unless otherwise indicated

up rate was $83.5 \%$. Table 2 displays the results of preoperative functional and radiological evaluation. Figures 2, 3, and 4 show the postoperative CT and Merchantview radiographs of one of our patients; there is normal congruence angle and patellar tilt angle and restoration of patellofemoral tracking. The mean Beighton score was 3.8 (range, 0-9); mean Insall-Salvati ratio was $1.3 \pm$ 0.2 (range, 0.8-1.8); mean $\mathrm{Q}$ angle was $14.5 \pm 3.5^{\circ}$ (range, $5-28^{\circ}$ ); and mean TT-TG value was $15.6 \pm 1.8$ $\mathrm{mm}$ (range, $8-23 \mathrm{~mm}$ ). As Table 3 shows, there was no relationship between postoperative patient-reported outcomes and the Beighton score, Insall-Salvati ratio, or TT-TG distance. The Q angle was negatively correlated to the Kujala score and the Lysholm score $(r$ value $=-$ $0.421, p=.009)$. Severity of trochlear dysplasia was classified according to Dejour (types A-D). Type A trochlear dysplasia was seen in 41 patients, type B in 20 patients, type $\mathrm{C}$ in 12 patients, and type $\mathrm{D}$ in 7 patients. The severity of trochlear dysplasia was not associated with postoperative patient-reported outcomes. Table 4 shows the patient-reported outcomes and satisfaction degree. At final follow-up, the mean Kujala score had improved from $69.4 \pm 7.9$ before surgery to $96.1 \pm 1.9$,

Table 2 Preoperative functional and radiographic evaluation

\begin{tabular}{ll}
\hline & Value \\
\hline Q angle & $14.5 \pm 3.5^{\circ}\left(5-28^{\circ}\right)$ \\
TT-TG distance, mm & $15.6 \pm 1.8(8-23)$ \\
Insall-Salvati ratio & $1.3 \pm 0.2(0.8-1.8)$ \\
Beighton score & $3.8 \pm 2.6(0-9)$ \\
Dejour trochlear dysplasia, $n(\%)$ & \\
Type A & $41(51.3)$ \\
Type B & $20(25.0)$ \\
Type C & $12(15.0)$ \\
Type D & $7(8.8)$ \\
\hline
\end{tabular}

Values are shown as mean \pm SD (range) unless otherwise indicated the Tegner score from $3.1 \pm 1.3$ to $5.9 \pm 1.3$, and the Lysholm score from $73.5 \pm 14.6$ to $95.3 \pm 3.4$. All scores demonstrated significant improvement $(P<.001)$. The outcome of the operation was graded as "excellent" by $57(86.4 \%)$ patients, as "good" by eight $(12.1 \%)$ patients, and as "fair" by one (1.5\%) patient. Two patients experienced recurrent patellar dislocations. One was a young man who suffered a knee injury while exercising 8 months after surgery. He refused revision surgery after manipulative reduction. He gave up strenuous exercise and has not suffered another dislocation so far. The other patient was a young woman who sprained her knee joint and then suffered re-dislocation while going down the stairs 1 year after surgery. One month after the re-dislocation, she underwent tibial tubercle osteotomy with revision MPFL repair. One year after the revision surgery, she returned to normal activity and currently experiences only occasional pain when going up and down the stairs. No infections, effusion, or screw site pain were encountered during follow-up.

\section{Discussion}

The mid-term clinical outcomes show that MPFL reconstruction using autologous gracilis tendon graft and the double-patellar tunnel technique under arthroscopy appears to be a reliable and safe method for treating recurrent patellar dislocation. The Kujala score, the Tegner score, and the Lysholm score demonstrated significant improvement. Postoperative patient-reported outcome was not associated with Beighton score, Insall-Salvati ratio, TT-TG distance, or severity of trochlear dysplasia. $\mathrm{Q}$ angle was negatively correlated to Kujala scores and Lysholm scores.

MPFL is the main structure preventing lateral dislocation of the patella $[28,29]$, and therefore patellar dislocation is invariably associated with MPFL rupture [30, 31]. MPFL is composed of double functional bundles, including an inferior-straight bundle at the medial aspect of the patella and a superior-oblique bundle at the superiormedial aspect of patella [12]. The patellar double-tunnel technique is recommended by many authors as the best method for achieving anatomical reconstruction [13, 3234]. The improvement in Kujala scores are reported to be similar to that achieved with other operative techniques $[32,35,36]$. In the patellar double-tunnel technique, two parallel tunnels are drilled through the patella from the medial margin to the lateral margin of the patella. In previous studies, the diameters of the bone tunnels have ranged from 3.2 to $4.5 \mathrm{~mm}[9,32,37,38]$. The existence of the patellar tunnels damages patellar integrity and strength, and there are several reports of patellar fracture occurring after MPFL reconstruction [9, 39]. Some surgeons therefore avoid the patellar tunnel technique and prefer to use a beltline rivet at the patellar inner margin to 


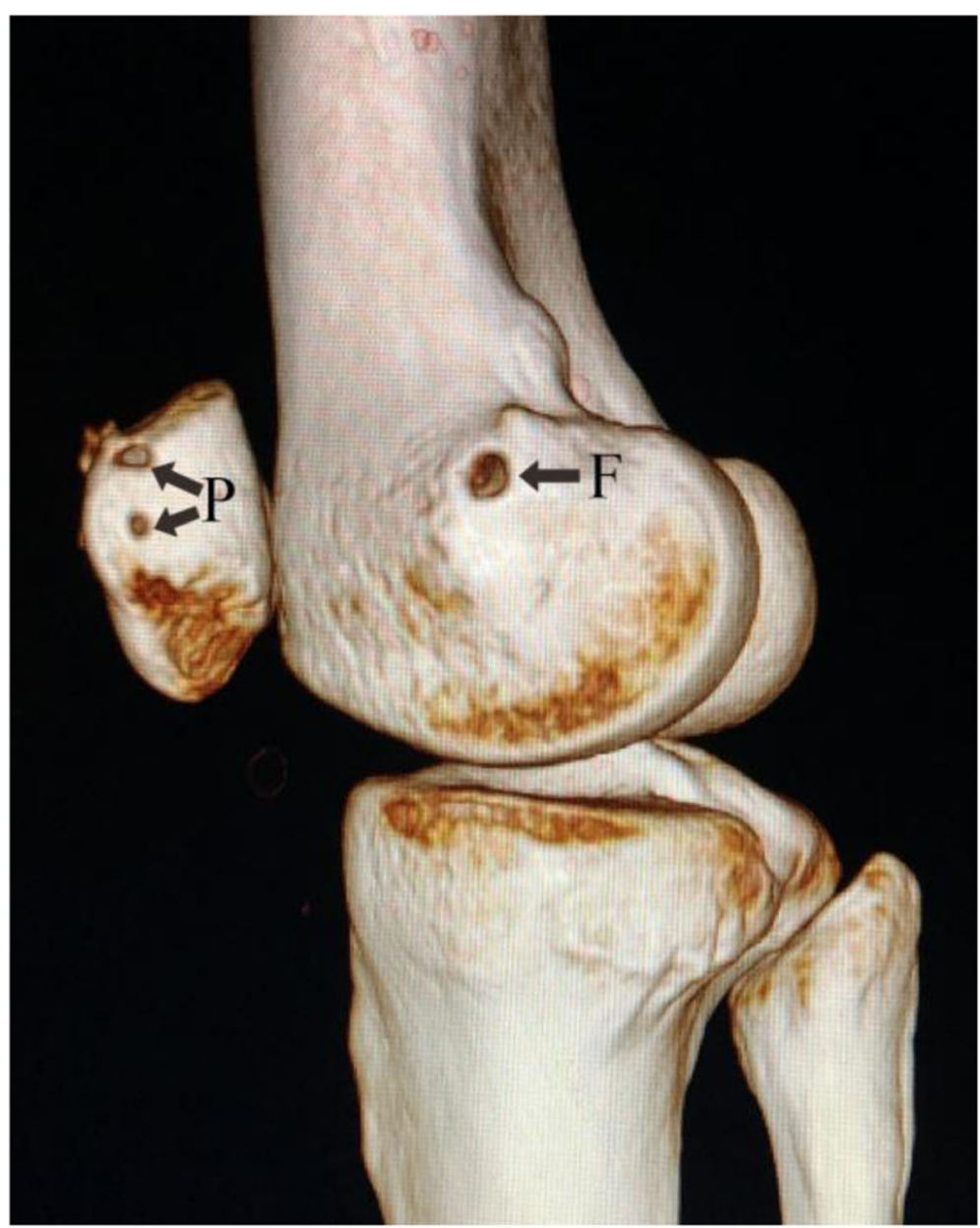

Fig. 2 Postoperative three-dimensional CT showing the position of the bone tunnels. F femoral bone tunnel, $P$ patellar bone tunnels

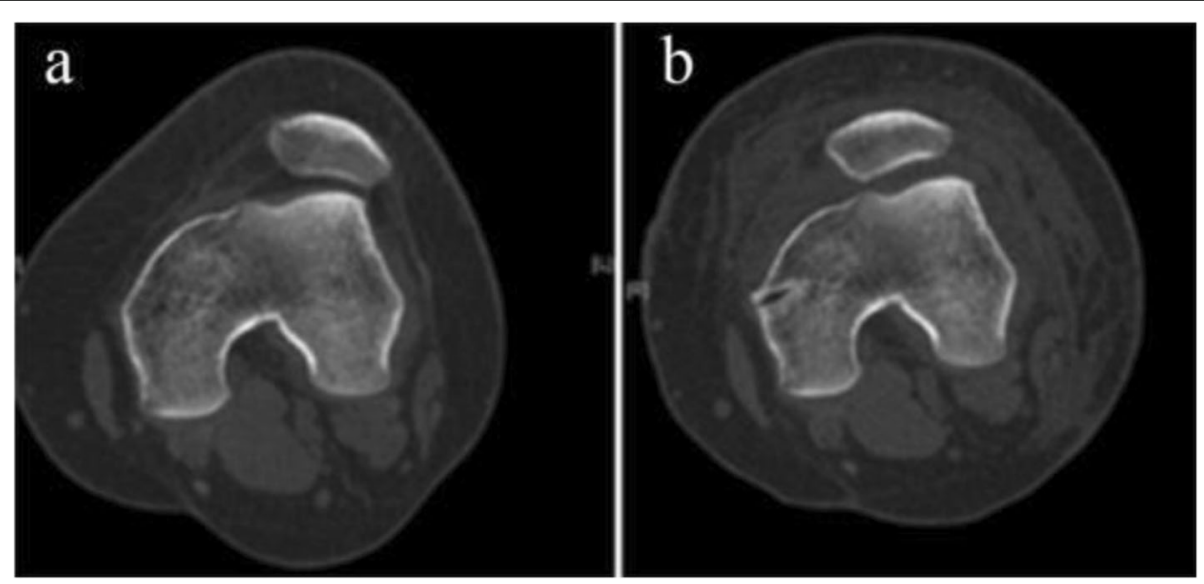

Fig. 3 Comparison of preoperative (a) and postoperative (b) CT scans demonstrating restoration of patellofemoral tracking 


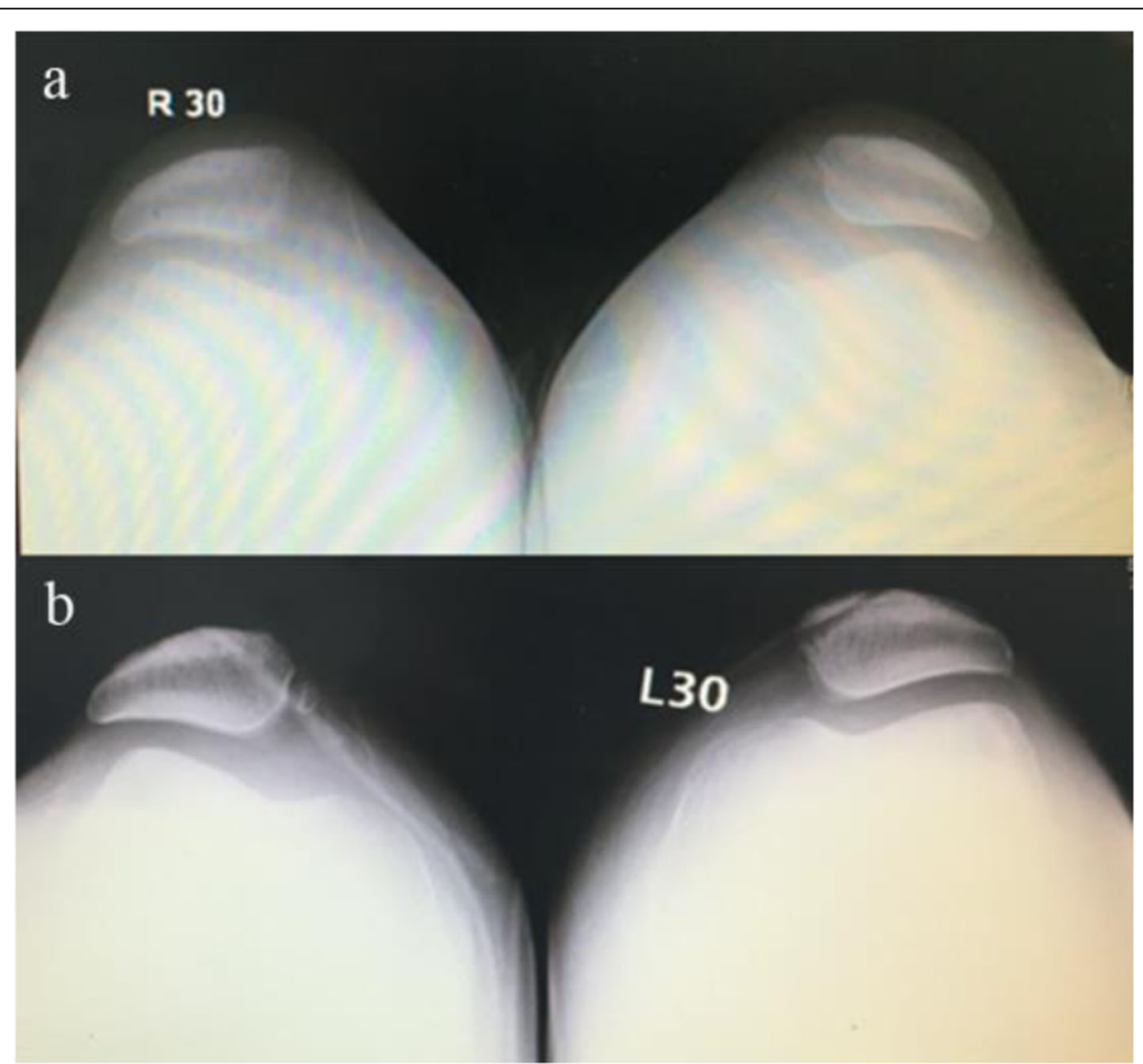

Fig. 4 Comparison of preoperative (a) and postoperative (b) Merchant-view radiographs demonstrating restoration of patellofemoral tracking

fix the graft [27, 40, 41]. However, the fixation strength of the beltline rivet is reported to be less than the strength of the bone tunnel tendon loop [42]. Reconstruction using suture anchors, which is another option, could also cause patellar fracture [43]. Studies comparing the common graft fixation methods-i.e., patellar bone tunnel fixation, beltline rivet fixation, direct suture fixation, and bone tunnel fixation without penetrating through the cortex-have found that only patellar bone tunnel fixation provides strength comparable to that of the undamaged MPFL. The strengths of beltline rivet and direct suture are particularly low [42]. We prefer to use the modified doublebone tunnel method. To prevent damage to patellar

Table 3 Relationship between postoperative patient-reported outcomes and Beighton score, Insall-Salvati ratio, Q angle, and Tा-TG distance

\begin{tabular}{|c|c|c|c|c|c|c|}
\hline & \multicolumn{2}{|c|}{ Kujala score } & \multicolumn{2}{|c|}{ Tegner score } & \multicolumn{2}{|c|}{ Lysholm score } \\
\hline & r value & $P$ value & r value & $P$ value & $r$ value & $P$ value \\
\hline Q-angle & -0.421 & 0.009 & -0.202 & 0.223 & -0.413 & .010 \\
\hline TT-TG distance & -0.112 & 0.502 & -0.096 & 0.565 & -0.159 & .342 \\
\hline Insall-Salvati ratio & -0.131 & 0.433 & -0.107 & 0.523 & -0.089 & .597 \\
\hline Beighton score & -0.017 & 0.918 & 0.034 & 0.841 & -0.077 & .645 \\
\hline
\end{tabular}

integrity, we restrict the diameter of the patellar tunnels to $3.5 \mathrm{~mm}$ and drill the bone tunnel from the inner lower patellar margin to the lateral upper margin so that the bone tunnel outlet is at the medial half of the patellar surface. We used this technique in all patients in this study and encountered no patellar fractures over the 5-year follow-up period.

The conventional method of reconstructing the MPFL requires two incisions to be made: one at the patellar inner margin and another at the femoral medial epicondyle. The extensor apparatus is incised to reach the second joint capsule layer and expose the MPFL. This

Table 4 Patient-reported outcomes and satisfaction degree

\begin{tabular}{llll}
\hline & Preoperative & Postoperative & $P$ value \\
\hline Kujala score & $69.4 \pm 7.9$ & $96.1 \pm 1.9$ & $P<.001$ \\
Tegner score & $3.1 \pm 1.3$ & $5.9 \pm 1.3$ & $P<.001$ \\
Lysholm score & $73.5 \pm 14.6$ & $95.3 \pm 3.4$ & $P<.001$ \\
Subjective satisfaction, $n(\%)$ & & \\
Excellent & $57(86.4)$ & \\
Good & $8(12.1)$ & \\
Fair & $1(1.5)$ & \\
\hline
\end{tabular}

Values are shown as mean \pm SD unless otherwise indicated 
additional surgical trauma and damage to the extensor apparatus is avoided by our method, where we use endoscopic fenestration of the articular capsule to expose the medial patellar bone margin and drill the bone tunnels under arthroscopy to reconstruct the MPFL. Trochlear dysplasia was found in every patient in our study. Some researchers have performed femoral trochleoplasty to correct trochlear dysplasia and improve patellar stability. Wagner et al. [44] suggested that severe trochlear dysplasia was related to poorer outcomes following MPFL reconstruction. Kohn et al. [45] concluded that severe trochlear dysplasia was an indication for combining a trochleoplasty with MPFL reconstruction; however, their study was only a case series and did not have a comparison group. Some studies reported that combining trochleoplasty with MPFL reconstruction causes increase in patellofemoral joint pressure and leads to pain and patellofemoral joint adhesion, seriously affecting postoperative outcomes [46, 47]. However, some recent studies have shown that trochleoplasty can provide good clinical results $[48,49]$. In the present study, for patients with arthrochalasis, patellar alta, trochlear dysplasia, and excessively large TT-TG, only simple MPFL reconstruction and lateral retinaculum release were performed. Although the abovementioned high-risk anatomical factors were not corrected, postoperative outcomes were still satisfactory. In fact, we found that postoperative outcome is not affected by these high-risk factors (other than the $\mathrm{Q}$ angle), which is in line with earlier reports $[19,36,50,51]$.

The $\mathrm{Q}$ angle was found to be negatively correlated to the Kujala score and the Lysholm score in this study. A large $\mathrm{Q}$ angle causes greater lateralization force on the patella, which increases the retropatellar pressure between the lateral facet of the patella and the lateral femoral condyle. This may explain the negative correlation between the $\mathrm{Q}$ angle and patient-reported outcomes. Our operation mainly aimed to restore the anatomy of the affected knee to the status before the first dislocation and thus prevent recurrent patellar dislocation. Restoration of anatomy and reconstruction of the MPFL are probably most beneficial for patients with MPFL rupture caused by trauma and congenitally weak medial structures. However, we recognize that some patients may still be at high risk for patellar dislocation even after MPFL reconstruction.

This study has several limitations. First, this was a retrospective study with a small sample. Second, it was a single-center, single-surgeon study; the results may not reflect the experience at other centers. Third, all patients did not undergo postoperative imaging follow-up. Fourth, a control group was not included. Given the difficulty in obtaining adequate films and the variable interpretation of these images, the use of the four-grade Dejour classification to evaluate trochlear dysplasia can also be considered a limitation of this study $[52,53]$. We have tried our best to obtain adequate films and maybe we can use CT or MRI to evaluate trochlear dysplasia in later studies.

\section{Conclusion}

MPFL reconstruction using autologous gracilis tendon graft and the double patellar tunnel technique under arthroscopy appears to be a reliable and safe method for treating recurrent patellar dislocation. The mid-term clinical outcomes in our sample were good.

\section{Abbreviation}

MPFL: Medial patellofemoral ligament

\section{Acknowledgments}

Not applicable,

\section{Authors' contributions}

GG performed the study. YX and PL conceived of the study and participated in its design. GG and YX were responsible for coordination, data collection/ interpretation, and proofreading of the final manuscript. All authors read and approved the final manuscript.

\section{Funding}

This study was funded by the National Natural Science Foundation of China (NFSC; grants 81672182 and 81871761), and Beijing New-star Plan of Science and Technology (xxjc201711).

\section{Availability of data and materials Not applicable.}

\section{Ethics approval and consent to participate}

The Ethics Committee of the Third Hospital of Peking University approved this study. (NO. 000067612014205). All subjects provided informed consent to take part in the study.

Consent for publication

Not applicable.

\section{Competing interests}

The authors declare that they have no competing interests.

Received: 10 July 2019 Accepted: 14 January 2020

Published online: 22 January 2020

\section{References}

1. Panni AS, Vasso M, Cerciello S. Acute patellar dislocation. What to do? Knee Surg Sports Traumatol Arthrosc. 2013;21:275-8.

2. Calapodopulos CJ, Nogueira MC, Eustáquio JMJ, Calapodopulos Júnior CJ, Rodrigues OA. Reconstruction of the medial patellofemoral ligament using autologous graft from quadriceps tendon to treat recurrent patellar dislocation. Rev Bras Ortop. 2016;51:187-93.

3. Mehta VM, Inoue M, Nomura E, Fithian DC. An algorithm guiding the evaluation and treatment of acute primary patellar dislocations. Sports Med Arthrosc. 2007;15:78-81.

4. Gravesen KS, Kallemose T, Blønd L, Troelsen A, Barfod KW. High incidence of acute and recurrent patellar dislocations: a retrospective nationwide epidemiological study involving 24.154 primary dislocations. Knee Surg Sports Traumatol Arthrosc. 2017;26:1204-9.

5. Carson WG Jr, James SL, Larson RL, Singer KM, Winternitz WW. Patellofemoral disorders: physical and radiographic evaluation. Part II: radiographic examination. Clin Orthop Relat Res. 1984:178-86.

6. Askenberger M, Janarv PM, Finnbogason T, Arendt EA. Morphology and anatomic patellar instability risk factors in first-time traumatic lateral patellar dislocations: a prospective magnetic resonance imaging study in skeletally immature children. Am J Sports Med. 2016. https://doi.org/10.1177/0363546516663498.

7. Lewallen L, Mcintosh A, Dahm D. First-time patellofemoral dislocation: risk factors for recurrent instability. J Knee Surg. 2015;28:303-10.

8. Steensen RN, Bentley JC, Trinh TQ, Backes JR, Wiltfong RE. The prevalence and combined prevalences of anatomic factors associated with recurrent 
patellar dislocation: a magnetic resonance imaging study. Am J Sports Med. 2015:43:921-7.

9. Shah JN, Howard JS, Flanigan DC, Brophy RH, Carey JL, Lattermann C. A systematic review of complications and failures associated with medial patellofemoral ligament reconstruction for recurrent patellar dislocation. Am J Sports Med. 2012:40:1916-23.

10. Kang H, Wang F, Cao J, Liu X, Ji G. A prospective randomized trial evaluating two different tensioning techniques for medial patellofemoral ligament reconstruction. Knee. 2016;23:826-9.

11. McNeilan RJ, Everhart JS, Mescher PK, Abouljoud M, Magnussen RA, Flanigan DC. Graft choice in isolated medial patellofemoral ligament reconstruction: a systematic review with meta-analysis of rates of recurrent instability and patient-reported outcomes for autograft, allograft, and synthetic options. Arthroscopy. 2018;34:1340-54.

12. Kang HJ, Wang F, Chen BC, Su YL, Zhang ZC, Yan CB. Functional bundles of the medial patellofemoral ligament. Knee Surg Sports Traumatol Arthrosc. 2010;18:1511-6.

13. Toritsuka Y, Amano H, Mae T, Uchida R, Hamada M, Ohzono K, et al. Dual tunnel medial patellofemoral ligament reconstruction for patients with patellar dislocation using a semitendinosus tendon autograft. Knee. 2011;18:214-9.

14. Schneider DK, Grawe B, Magnussen RA, Ceasar A, Parikh SN, Wall EJ, et al. Outcomes after isolated medial patellofemoral ligament reconstruction for the treatment of recurrent lateral patellar dislocations. Am J Sports Med. 2016;44:2993-3005.

15. Fisher B, Nyland J, Brand E, Curtin B. Medial patellofemoral ligament reconstruction for recurrent patellar dislocation: a systematic review including rehabilitation and return-to-sports efficacy. Arthroscopy. 2010;26:1384-94.

16. Howells NR, Eldridge JD. Medial patellofemoral ligament reconstruction for patellar instability in patients with hypermobility: a case control study. J Bone Joint Surg Br. 2012;94:1655-9.

17. Dejour H, Walch G, Nove-Josserand L, Guier C. Factors of patellar instability: an anatomic radiographic study. Knee Surg Sports Traumatol Arthrosc. 1994;2:19-26.

18. Bicos J, Fulkerson JP, Amis A. Current concepts review: the medial patellofemoral ligament. Am J Sports Med. 2007;35:484-92.

19. Schottle PB, Fucentese SF, Romero J. Clinical and radiological outcome of medial patellofemoral ligament reconstruction with a semitendinosus autograft for patella instability. Knee Surg Sports Traumatol Arthrosc. 2005; 13:516-21.

20. Kuroda R, Kambic H, Valdevit A, Andrish JT. Articular cartilage contact pressure after tibial tuberosity transfer. A cadaveric study. Am J Sports Med. 2001;29:403-9.

21. Horton MG, Hall TL. Quadriceps femoris muscle angle: normal values and relationships with gender and selected skeletal measures. Phys Ther. 1989; 69:897-901.

22. Insall J, Salvati E. Patella position in the normal knee joint. Radiology. 1971; 101:101-4.

23. Amis AA, Firer P, Mountney J, Senavongse W, Thomas NP. Anatomy and biomechanics of the medial patellofemoral ligament. Knee. 2003;10:215-20.

24. Nomura $\mathrm{E}$, Inoue $\mathrm{M}$, Osada N. Anatomical analysis of the medial patellofemoral ligament of the knee, especially the femoral attachment. Knee Surg Sports Traumatol Arthrosc. 2005;13:510-5.

25. Deie M, Ochi M, Adachi N, Shibuya H, Nakamae A. Medial patellofemoral ligament reconstruction fixed with a cylindrical bone plug and a grafted semitendinosus tendon at the original femoral site for recurrent patellar dislocation. Am J Sports Med. 2011;39:140-5.

26. Redziniak DE, Diduch DR, Mihalko WM, Fulkerson JP, Novicoff WM, SheibaniRad S, et al. Patellar instability. J Bone Joint Surg Am. 2009;91:2264-75.

27. Schottle PB, Hensler D, Imhoff AB. Anatomical double-bundle MPFL reconstruction with an aperture fixation. Knee Surg Sports Traumatol Arthrosc. 2010;18:147-51.

28. Zaffagnini S, Colle F, Lopomo N, Sharma B, Bignozzi S, Dejour D, et al. The influence of medial patellofemoral ligament on patellofemoral joint kinematics and patellar stability. Knee Surg Sports Traumatol Arthrosc. 2013; 21:2164-71.

29. Nomura E, Horiuchi Y, Kihara M. Medial patellofemoral ligament restraint in lateral patellar translation and reconstruction. Knee. 2000;7:121-7.

30. Sillanpaa P, Mattila VM, livonen T, Visuri T, Pihlajamaki H. Incidence and risk factors of acute traumatic primary patellar dislocation. Med Sci Sports Exerc. 2008;40:606-11.

31. Sallay PI, Poggi J, Speer KP, Garrett WE. Acute dislocation of the patella. A correlative pathoanatomic study. Am J Sports Med. 1996;24:52-60.
32. Christiansen SE, Jacobsen BW, Lund B, Lind M. Reconstruction of the medial patellofemoral ligament with gracilis tendon autograft in transverse patellar drill holes. Arthroscopy. 2008;24:82-7.

33. Ronga M, Oliva F, Longo UG, Testa V, Capasso G, Maffulli N. Isolated medial patellofemoral ligament reconstruction for recurrent patellar dislocation. Am J Sports Med. 2009;37:1735-42.

34. Lippacher S, Dreyhaupt J, Williams SR, Reichel H, Nelitz M. Reconstruction of the medial patellofemoral ligament: clinical outcomes and return to sports. Am J Sports Med. 2014;42:1661-8.

35. Sillanpaa P, Mattila VM, Visuri T, Maenpaa H, Pihlajamaki H. Ligament reconstruction versus distal realignment for patellar dislocation. Clin Orthop Relat Res. 2008;466:1475-84.

36. Steiner TM, Torga-Spak R, Teitge RA. Medial patellofemoral ligament reconstruction in patients with lateral patellar instability and trochlear dysplasia. Am J Sports Med. 2006;34:1254-61.

37. Maeno S, Hashimoto D, Otani T, Masumoto K, Fukui Y, Nishiyama M, et al. Medial patellofemoral ligament reconstruction with hanger lifting procedure. Knee Surg Sports Traumatol Arthrosc. 2010;18:157-60.

38. Panni AS, Alam M, Cerciello S, Vasso M, Maffulli N. Medial patellofemoral ligament reconstruction with a divergent patellar transverse 2-tunnel technique. Am J Sports Med. 2011;39:2647-55.

39. Schiphouwer L, Rood A, Tigchelaar S, Koëter S. Complications of medial patellofemoral ligament reconstruction using two transverse patellar tunnels. Knee Surg Sports Traumatol Arthrosc. 2016;25:245-50.

40. Schottle PB, Romero J, Schmeling A, Weiler A. Technical note: anatomical reconstruction of the medial patellofemoral ligament using a free gracilis autograft. Arch Orthop Trauma Surg. 2008;128:479-84.

41. Kim TS, Kim HJ, Ra IH, Kyung HS. Medial Patellofemoral ligament reconstruction for recurrent patellar instability using a gracilis autograft without bone tunnel. Clin Orthop Surg. 2015;7:457-64.

42. Mountney J, Senavongse W, Amis AA, Thomas NP. Tensile strength of the medial patellofemoral ligament before and after repair or reconstruction. J Bone Joint Surg Br. 2005;87:36-40.

43. Dhinsa BS, Bhamra JS, James C, Dunnet W, Zahn H. Patella fracture after medial patellofemoral ligament reconstruction using suture anchors. Knee. 2013;20:605-8.

44. Wagner D, Pfalzer F, Hingelbaum S, Huth J, Mauch F, Bauer G. The influence of risk factors on clinical outcomes following anatomical medial patellofemoral ligament (MPFL) reconstruction using the gracilis tendon. Knee Surg Sports Traumatol Arthrosc. 2013;21:318-24.

45. Kohn LM, Meidinger G, Beitzel K, Banke IJ, Hensler D, Imhoff AB, et al. Isolated and combined medial patellofemoral ligament reconstruction in revision surgery for patellofemoral instability: a prospective study. Am J Sports Med. 2013;41:2128-35.

46. Dejour D, Le Coultre B. Osteotomies in patello-femoral instabilities. Sports Med Arthrosc. 2007;15:39-46.

47. Verdonk R, Jansegers E, Stuyts B. Trochleoplasty in dysplastic knee trochlea. Knee Surg Sports Traumatol Arthrosc. 2005;13:529-33.

48. Wind RJP, Heesterbeek PJC, Wymenga AB. A combined procedure with Bereiter-type trochleoplasty leads to a stable patellofemoral joint at 5-year follow-up. Knee Surg Sports Traumatol Arthrosc. 2019;27:716-23.

49. Longo UG, Vincenzo C, Mannering N, Ciuffreda M, Salvatore G, Berton A, et al. Trochleoplasty techniques provide good clinical results in patients with trochlear dysplasia. Knee Surg Sports Traumatol Arthrosc. 2017:1-19.

50. Howells NR, Barnett AJ, Ahearn N, Ansari A, Eldridge JD. Medial patellofemoral ligament reconstruction: a prospective outcome assessment of a large single centre series. J Bone Joint Surg Br. 2012;94:1202-8.

51. Feller JA, Richmond AK, Wasiak J. Medial patellofemoral ligament reconstruction as an isolated or combined procedure for recurrent patellar instability. Knee Surg Sports Traumatol Arthrosc. 2014;22:2470-6.

52. Stepanovich M, Bomar JD, Pennock AT. Are the current classifications and radiographic measurements for trochlear dysplasia appropriate in the skeletally immature patient? Orthop J Sports Med. 2016;4(10):23259671 16669490.

53. Lippacher S, Dejour D, Elsharkawi M, Dornacher D, Ring C, Dreyhaupt J, et al. Observer agreement on the Dejour trochlear dysplasia classification: a comparison of true lateral radiographs and axial magnetic resonance images. Am J Sports Med. 2012;40:837-43.

\section{Publisher's Note}

Springer Nature remains neutral with regard to jurisdictional claims in published maps and institutional affiliations. 